\title{
UCRL-TR-208744
}

LAWRENCE LIVERMORE N A T IO N A L LABORATORY

\section{Error Sources in the ETA Energy Analyzer Measurement}

W. E. Nexsen

December 22, 2004 
This document was prepared as an account of work sponsored by an agency of the United States Government. Neither the United States Government nor the University of California nor any of their employees, makes any warranty, express or implied, or assumes any legal liability or responsibility for the accuracy, completeness, or usefulness of any information, apparatus, product, or process disclosed, or represents that its use would not infringe privately owned rights. Reference herein to any specific commercial product, process, or service by trade name, trademark, manufacturer, or otherwise, does not necessarily constitute or imply its endorsement, recommendation, or favoring by the United States Government or the University of California. The views and opinions of authors expressed herein do not necessarily state or reflect those of the United States Government or the University of California, and shall not be used for advertising or product endorsement purposes.

This work was performed under the auspices of the U.S. Department of Energy by University of California, Lawrence Livermore National Laboratory under Contract W-7405-Eng-48. 
(Final draft 12-20-04)

\title{
Error Sources in the ETA Energy Analyzer Measurement W.E. Nexsen
}

\begin{abstract}
At present the ETA beam energy as measured by the ETA energy analyzer and the DARHT spectrometer differ by $\sim 12 \%$. This discrepancy is due to two sources, an overestimate of the effective length of the ETA energy analyzer bending-field, and data reduction methods that are not valid. The discrepancy can be eliminated if we return to the original process of measuring the angular deflection of the beam and use a value of $43.2 \mathrm{~cm}$ for the effective length of the axial field profile.
\end{abstract}

Introduction: ETA beam energy measurements taken with the ETA energy analyzer have shown a nominal value of $\sim 5.8 \mathrm{MeV}$, however, the FITS beam model gave a best fit to initial conditions (energy, current, emittance, envelope radius, and envelope slope) for $\sim 5.0 \mathrm{MeV} .{ }^{1}$ This lower value was corroborated by measurements taken with an analyzer that measures the momentum of beam electrons scattered at small angles by a fine wire. ${ }^{2}$ To resolve this discrepancy, the DARHT spectrometer was brought from LANL to measure the beam energy under the same conditions on the same day as a set of ETA analyzer measurements. The DARHT spectrometer has been calibrated with an ion beam of known energy and can be assumed to give an absolute measurement that is correct within about a percent. Although simultaneous measurements could not be made, it was assumed that operation was stable enough that consecutive shots would be comparable. Glen Westenskow ${ }^{3}$, in summarizing the results, reported that the ETA energy analyzer measurements appeared to be $\sim 12 \%$ higher than those of the DARHT spectrometer, consistent with the previous observations. I have identified two items that when compounded appear to explain the measurement difference.

The ETA energy analyzer: The ETA analyzer is located in the beam transport section down stream of the accelerator exit. It employs a magnetic field normal to the ETA axis to bend the total beam into a side arm whose axis is at $45^{0}$ to the ETA axis. A pair of beam position monitors (BPM EA1 and BPM EA2) separated by a $0.86 \mathrm{~m}$ drift length are used to measure the angle, $\delta_{12}$, usually small, that the beam centroid makes with the ETA axis in the bending plane at the entrance to the analyzer while on the exit side arm another pair of beam position monitors (BPM EA3 and BPM EA4) separated by $0.25 \mathrm{~m}$ drift are used to measure the angle, $\delta_{34}$, that the deflected beam centroid makes with the side arm axis in the bending plane. The angle of deflection due to the bending field is

$$
\theta=\pi / 4+\delta_{34}-\sqrt{2} \delta_{12}
$$

The governing equation relating electron momentum to deflection by a magnetic field normal to the electron path is

${ }^{1}$ Frank Chambers and Brett Raymond, Private communication.

${ }^{2}$ Thomas J. Fessenden, The Astron On-Line Beam Energy Analyzer", RSI $\underline{43}, 1090$ (1972)

${ }^{3}$ Glen Westenskow, "Measurement of the ETA-II Beam Energy with the DAHRT Spectrometer", Internal memo, October, 2004. 


$$
\beta \gamma=\left(\frac{\mathrm{e}}{\mathrm{mc}^{2}}\right) \frac{\int_{-\infty}^{\infty} \mathrm{Bds}}{\theta}=\frac{\mathrm{B}_{\max } \int_{-\infty}^{\infty}\left(\mathrm{B}(\mathrm{s}) / \mathrm{B}_{\max }\right) \mathrm{ds}}{1705 \theta}=\frac{\mathrm{B}_{\max } \mathrm{L}_{\mathrm{eff}}}{1705 \theta}(\mathrm{cgs})
$$

$\mathrm{L}_{\text {eff }}$ is obtained by integrating the field profile along the path of the central orbit. The factor, $B_{\max } L_{\text {eff }} / \theta$, as a measure of the electron momentum, is independent of the profile of the bending field along the particle path through the bending field. For a coordinate system with the initial path along the z-axis, the field along the x-axis and the deflection in the $\mathrm{y}$-direction, we define $\mathrm{L}_{\mathrm{Z}}$ by

$$
\mathrm{L}_{\mathrm{z}} \equiv \int_{-\infty}^{\infty}\left(\mathrm{B}(\mathrm{s}) / \mathrm{B}_{\max }\right) \mathrm{dz} \text {. }
$$

$\mathrm{L}_{\text {eff }}$ is related to $\mathrm{L}_{\mathrm{Z}}$, the integral along the z-axis of the field profile along the particle path by

$$
\mathrm{L}_{\text {eff }}=\frac{\theta}{\sin \theta} \mathrm{L}_{\mathrm{z}}
$$

Combining Equations 2 and 3 yields

$$
\beta \gamma=\frac{B_{\max } L_{z}}{1705 \sin \theta}
$$

Whereas $\mathrm{L}_{\mathrm{eff}}$ is a function of the bending angle, $\mathrm{L}_{\mathrm{Z}}$ is assumed constant for values of $\delta_{34}$ small compared with $\pi / 4$ and when combined with measured values of $B_{\max }$ and $\theta$ in Equation 4 yields $\beta \gamma$ and thus the energy.

ETA analyzer magnetic field profile: The bending magnet poles are 10" long in the axial direction and 21" wide. The geometric center of the poles is offset 5" from the ETA axis. Because the beam must pass unperturbed through the analyzer when the bending field is off, the magnet has a 6.75" gap. Figure 1 shows the ETA energy analyzer bending-field profile along the z-axis. Two sets of measurements, an earlier set taken at $2 \mathrm{~mm}$ intervals but not extending very far into the fringing field ${ }^{4}$, and a later set taken along the machine axis at a coarser interval (1") but extending to very low values of the field ${ }^{5}$ have been combined to yield the profile data. Over the region of overlap the agreement between the two data sets was very good. Easily discernable is the depression of the field on axis on the entrance side by the ferrite core of the second beam bug.

The integral of the axial profile $(\mathrm{y}=0)$ was approximated by a trapezoidal integration of these tabulated values and their spacing. This yielded a value for $L_{z}$ of $44.3 \mathrm{~cm}$ that would be our final answer if we could disregard variation of the bending field in the $y$ direction. However due to the finite dimension of the magnet in the y-direction, the zprofile of the field along the particle orbit is less than the $y=0$ profile for large values of $z$, consequently we expect a smaller value for the $\mathrm{L}_{\mathrm{Z}}$ of this profile. The earlier mapping of the magnet which included also the values of $\mathrm{B}(\mathrm{y}, \mathrm{z})$ on a $20 \mathrm{~mm}$ grid allows us to better approximate the field along the particle path. The profile along the entrance side will be the same while along the exit side we use the field along the $45^{0}$ diagonal, $\mathrm{B}(\mathrm{y}=\mathrm{z}, \mathrm{z})$. This

${ }^{4}$ Roger Van Maren, 1989.

${ }^{5}$ John Weir, 2004. 
profile is designated by $\mathrm{y}=\mathrm{z}$ in Figure 1; ignoring the $\mathrm{y}$-variation is obviously a good approximation over most of the particle orbit. The fringing field was not measured along the diagonal; the dashed line was obtained by rotating the axial fringing field to lie

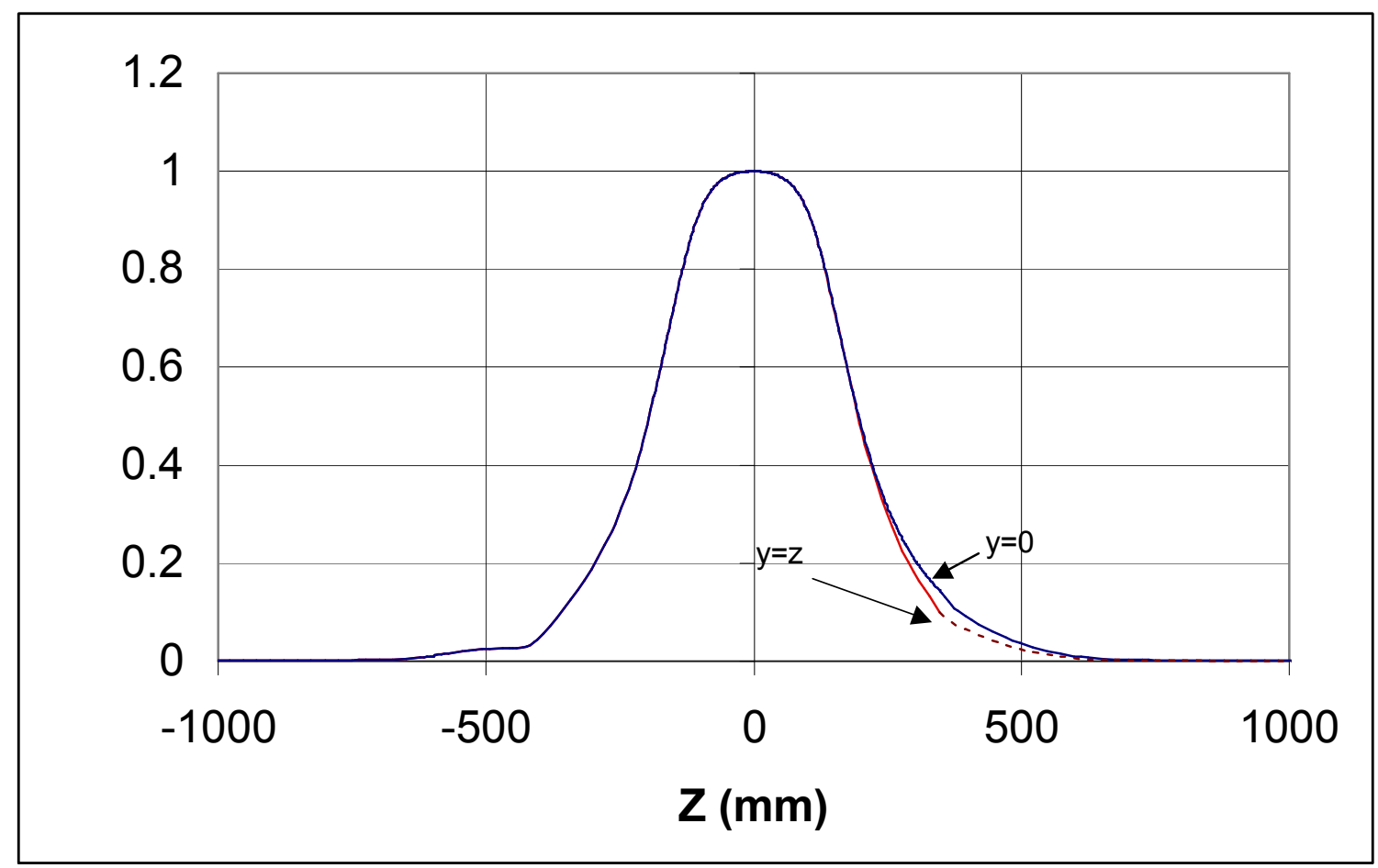

Figure 1 Bending field profile along particle path, axial profile overlaid.

along the diagonal, scaling to tie into the measured data and projecting the profile onto the z-axis, a crude but more or less reasonable approximation. This profile gives a $\mathrm{L}_{\mathrm{z}}$ of $43.5 \mathrm{~cm}$; the lower limit value is $42.3 \mathrm{~cm}$ if one disregards the contribution of the dashed portion of the profile while if one assumes that the z-variation of the fringing field has the same shape as the axial profile and only scales it to tie in to the measured value one obtains a value of $43.6 \mathrm{~cm}$. A value for $\mathrm{L}_{\mathrm{Z}}$ of $45.9 \mathrm{~cm}$ is used at present in the ETA datahandling program, Suicidetails, in reducing the analyzer data. If we take 43.5 as the best value for $\mathrm{L}_{\mathrm{Z}}$ we find that we are overestimating the momentum by $5.5 \%$ for this reason alone.

Data reduction: Normally, magnetic analyzers are designed with a gap between their poles that is small compared with other magnet dimensions and the field is essentially constant over a large fraction of the particle orbit. For this case a good approximation for calculating the transport of the beam through the magnet is to use the sharp cutoff fringing field (SCOFF) model of the bending field, i.e., the radius of curvature makes an abrupt change from infinite to a constant value at a virtual boundary on the entrance side and returns to infinite at a similar boundary on the exit side. From Figure 1 it is obvious that because the gap dimension is of the same order as the other magnet dimensions, only near the maximum is the field anywhere near constant, the radius of curvature, proportional to $1 / \mathrm{B}$, is not constant and the center of curvature not fixed in position as the 
beam passes through the magnet, consequently the sharp cutoff model is not a good approximation for the ETA energy analyzer field and the matrix transport equations which assume a constant value of bending radius with center fixed and which, at present, are used in Suicidetails are not applicable. There is no constraint that a beam entering the magnet on axis, after bending through $\pi / 4$ radian, will exit along the side arm axis. To illustrate this point I have calculated beam trajectories using the Figure $1 \mathrm{y}=\mathrm{z}$ profile and peak field value corresponding to that of the experimental data taken on the day that the DARHT spectrometer was mounted on ETA. It was assumed that the field profile was independent of the peak value over the range of interest. The experimental data was taken

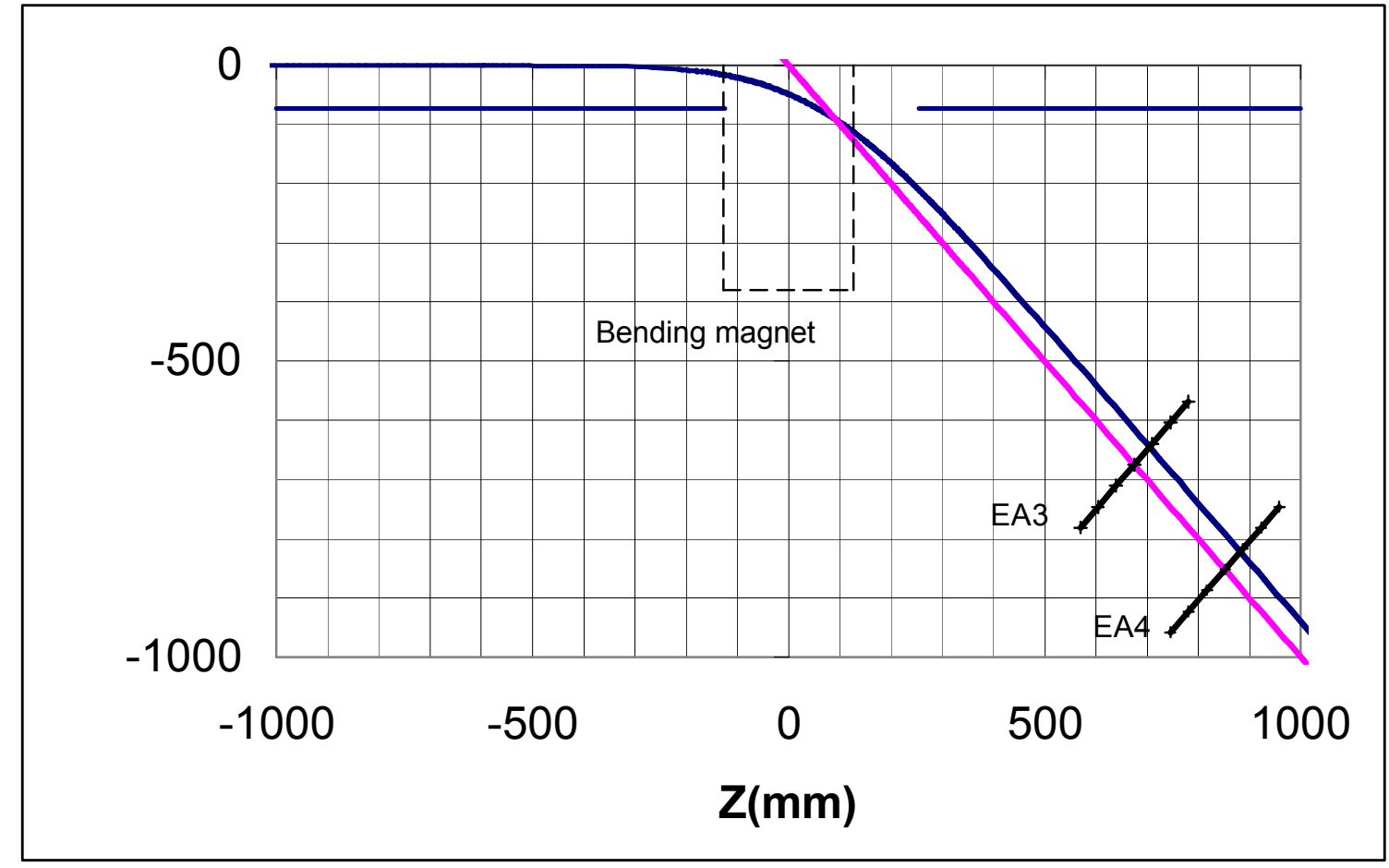

Figure 2 Trajectory of $5.49 \mathrm{MeV}$ electron in $325 \mathrm{G}$ peak field with Figure $1 \mathrm{y}=\mathrm{z}$ profile

for a magnet current of 47 A corresponding to a peak bending field of 325 Gauss. Figure 2 shows that in the measured field profile with this peak value a $5.49 \mathrm{MeV}$ particle will be deflected $\pi / 4$ radian. While parallel to the axis of the side arm the trajectory is almost $5 \mathrm{~cm}$ off axis.

Discussion: Originally the ETA analyzer used the two pairs of BPMs to measure the angular deflection of the beam and obtain $\beta \gamma$ from Equation 4. When ETA was reactivated after being mothballed for a number of years the sharp cutoff model transport equations were introduced into the analyzer data reduction software. When applicable, these allow the calculation of the energy using only three of the four BPM signals and lead to three additional ways of obtaining the energy value. When it was found that these values did not agree everyone adopted his favorite BPM combination. One persistent favorite was to use the two BPMs on the entrance side and the first on the exit side, while adjusting the bending field to center the beam on the latter. The trajectory of a 5.23 
$\mathrm{MeV}$ electron in the same field as Figure 2 is shown in Figure 3. Since the orbit passes through the center of the first BPM on the exit side, the sharp cutoff model would assume that the particle had been deflected through $\pi / 4(=0.78540)$ radian rather than the actual value of 0.84107 radian and would interpret the energy as $5.49 \mathrm{MeV}$, an over-estimate of $5 \%$.

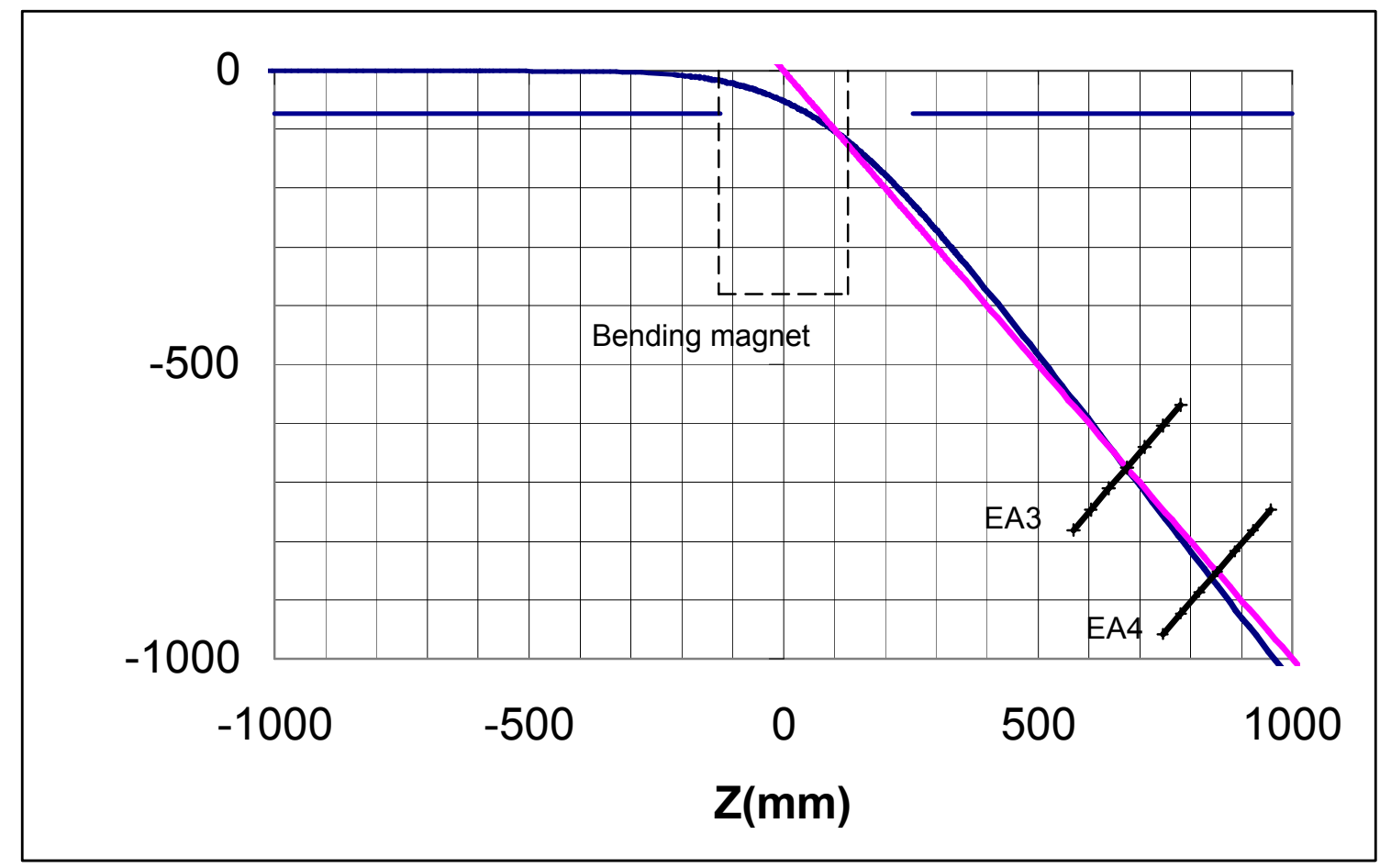

Figure 3 Trajectory of 5.23 MeV electron in field of Figure $1 \mathrm{y}=\mathrm{z}$ profile.

Using four beam bugs to measure the total angle of bend is the only legitimate approach to energy measurement with the ETA analyzer. On the day that the energy analyzer was compared with the DARHT spectrometer there is deflection data on BPM EA4 only on two shots $(4267 \# 34,35)$ early in the day. (Apparently the BPM was not connected for the remainder of the day.) These records have been time aligned and reduced using $\mathrm{L}_{\mathrm{Z}}=43.5 \mathrm{~cm}$. The smoothed results are shown in Figure 4. It is unfortunate that these are the only usable energy analyzer data shots as there appears to be a real difference between them. The closest usable DARHT spectrometer data records are from shots $4267 \# 37,38$. Their reduction is shown in Figure 5. By shots 37 and 38 operation has settled down as evidenced by how well they overlay. Overlaid is the 4267 $\# 34,35$ data re-reduced using a value for $\mathrm{L}_{\mathrm{Z}}$ of $43.2 \mathrm{~cm}$, a further reduction of $0.7 \%$. I don't know whether the deviation between the analyzer and spectrometer measurement at higher energy is real; the analyzer is showing an energy sweep of the order of $10 \%$, which is much more than that observed for best ETA operation. If the beam was centered on BPM EA3 at the energy minimum than for the energy maximum the centroid would be halfway to the wall at BPM EA4 and the BPM response nonlinear. Ideally, if we face such a large energy sweep the analyzer field should be adjusted so that the sweep is centered on BPM EA4. 


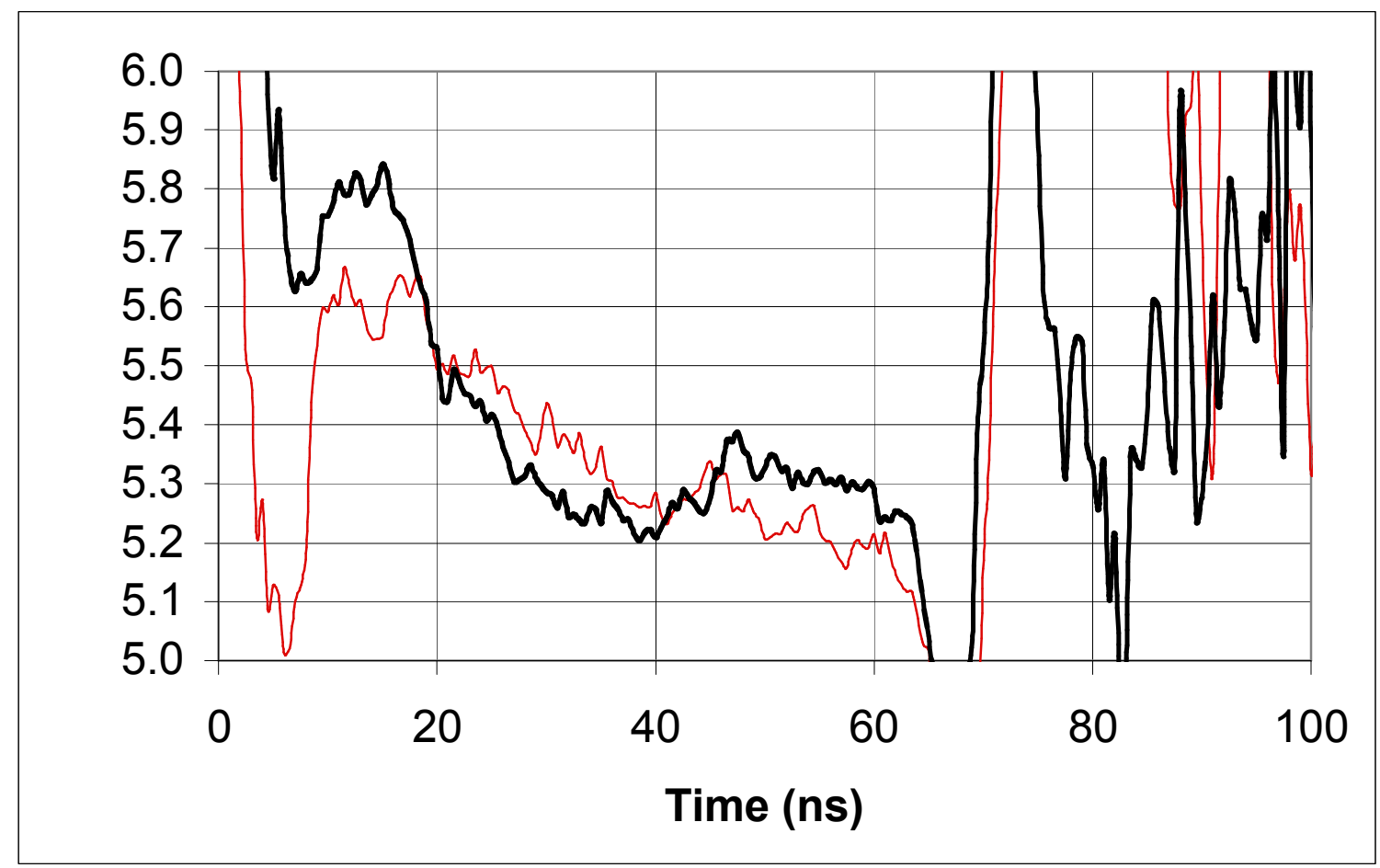

Figure 4 Beam kinetic energy for shots 4267 34,35 as measured by ETA energy analyzer ( reduced using $L_{z}=43.5 \mathrm{~cm}$ ).

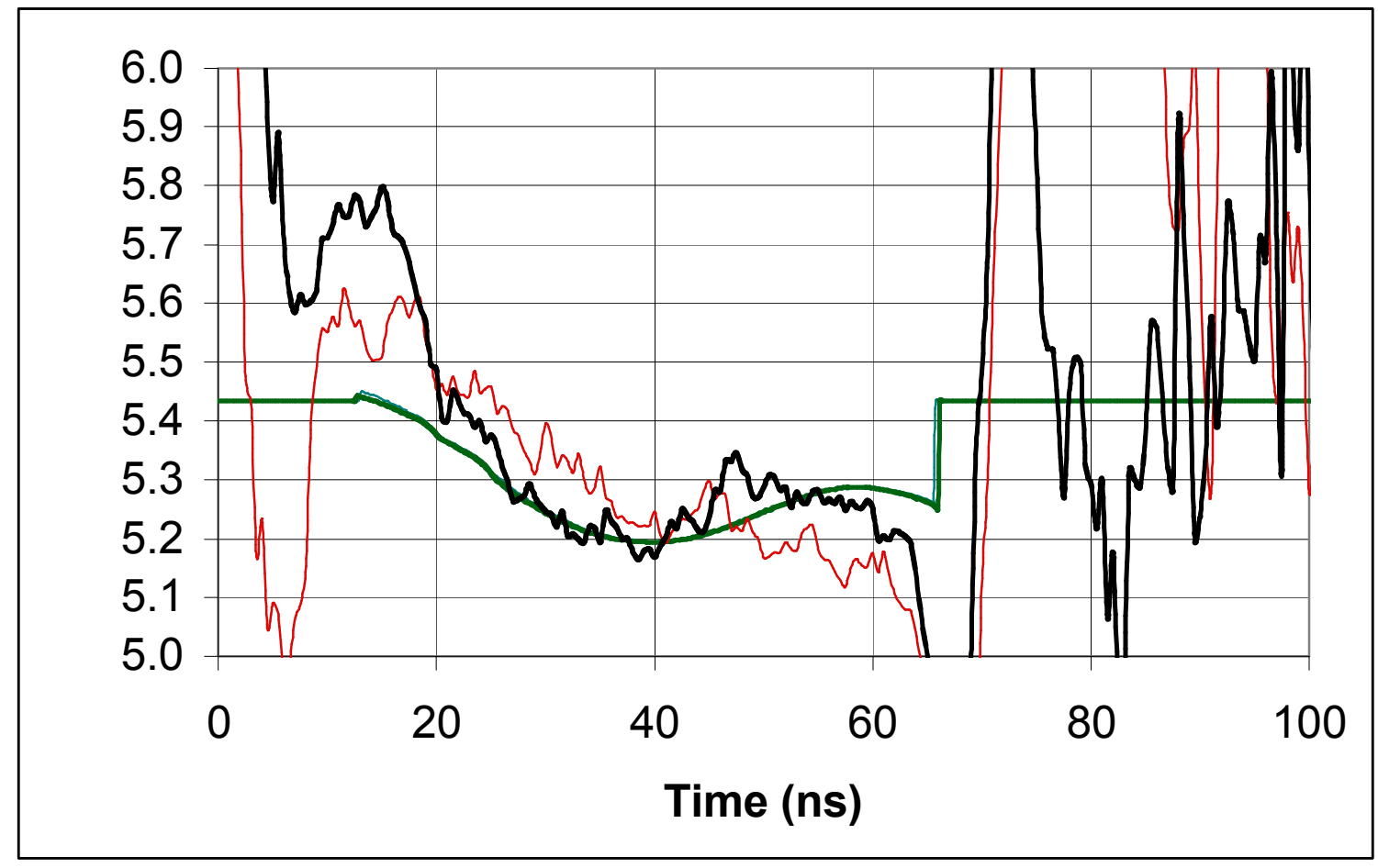

Figure 5 Beam kinetic energy for shots 426737,38 as measured by the DARHT spectrometer overlaid on data of Figure 4, re-reduced with $L_{z}=43.2 \mathrm{~cm}$. 
I am aware of two further questions that have been raised about the legitimacy of the analyzer measurement. The first of these concerns whether the analyzer rectangular cross-section vacuum chamber that is closely coupled to the first BPM on the exit side influences the return current distribution in the BPM foil and thus introduces an error in the position measurement. This does not appear to me to be likely since the return current must be distributed in the circular cross section beam bug so as to terminate the beam field. This field is almost a plane wave due to its relativistic contraction in the axial direction and consequently current flow patterns in adjacent regions will have little effect on the measurement. The second concerns the influence of the observed current loss of about $40 \%$ of the deflected beam on the energy measurement. Since the instantaneous energy spread is very small the instantaneous angular deflection of the beam particles will all be nearly the same, consequently while the centroid position may shift with current loss, the angular deflection of the centroid should be independent of the part of the beam cross section that is lost and measuring deflection angle to obtain beam energy should still be correct.

Conclusion: The discrepancy between the DARHT spectrometer and the ETA analyzer measurement of the ETA beam energy is primarily due to the compounding of two errors:1) the use of a longer axial profile length than that obtained from the measured field profile, 2) the use of the sharp cutoff model transport equations in the data reduction. Previous studies of the discrepancy ${ }^{6}$ have fastened on the value of $\mathrm{L}_{\mathrm{Z}}$ along the axis but all seem to arrive at values greater than that of $44.3 \mathrm{~cm}$ for reasons that I don't understand. The value of $\mathrm{L}_{\mathrm{Z}}=44.3 \mathrm{~cm}$ is consistent with the value of $44.8 \mathrm{~cm}$ originally used in the reduction and the value of $43.9 \mathrm{~cm}$ obtained from a Poisson calculation ${ }^{7}$. Apparently, until now, no one had reflected on the ramifications of the use of the sharp cutoff model transport equations. These equations, which assume a constant radius of curvature and a central orbit exit on the $45^{\circ}$ side arm axis, should not be used. By using a

value for $\mathrm{L}_{\mathrm{Z}}$ consistent with the measured field profile and the two pairs of BPMs to measure the angular deflection of the beam centroid, the disagreement between the ETA energy analyzer and the DARHT spectrometer is reduced to the order of $1 \%$. Ideally, with only two perhaps unrepresentative shots to compare the analyzer with the spectrometer, one would like to take more data. In the absence of such a replay I recommend that the DARHT spectrometer be assumed to be correct and, in the ETA energy analyzer data reduction, an effective axial of $\mathrm{L}_{\mathrm{Z}}=43.2 \mathrm{~cm}$ be used to give agreement with the spectrometer data.

This work was performed under the auspices of the U.S. Department of Energy by the University of California, Lawrence Livermore National Laboratory under Contract No. W-7405-Eng-48.

\footnotetext{
${ }^{6}$ Frank Chambers and Brett Raymond, Private communication.

${ }^{7}$ Art Paul, "ETAII Energy Analyzer Calibration", Internal memo EACALIB, 8/25/97.
} 